\title{
EXOTIC SPHERES AND JOHN MILNOR
}

\author{
MARCo ABATE \\ Dipartimento di Matematica \\ Università di Pisa \\ Largo Pontecorvo 5 \\ 56127 Pisa \\ Italia \\ Telefono: $+39 / 050 / 2213.230$ \\ Fax: $+39 / 050 / 2213.224$ \\ E-mail: abate@dm.unipi.it \\ Talk given in the Congress \\ "Matematica e Cultura 2012" \\ on March 31, 2012
}


There are mathematicians able to solve incredibly difficult problems devising amazingly new ideas. There are mathematicians with a sure grasp of entire subjects, able to single out the more promising research directions. There are mathematicians with a crystal clear vision, able to explain and clarify any subject they talk about. And then there is John Milnor. He is all three: he solves, understands and explains, at an exceptional level on all counts. And he is a nice guy too.

In this short note, after a brief bibliographical sketch, I shall try and describe one of the most famous theorems proved by Milnor. We shall not go very much beyond explaining what the statement means, but I hope it would be enough for getting at least an idea of the brilliance of Milnor's mathematics.

\section{John Willard Milnor}

John Willard Milnor (known as Jack by his friends and colleagues) was born on 20 February 1931 in Orange, New Jersey, USA. He published his first mathematical paper [1] when he was only 19 years old. And it was not just any paper: he solved a 20 years old problem on the differential geometry of knots in 3-dimensional space (see [2] for a very short introduction to knots), proving a theorem, the Milnor-Fáry theorem on the total curvature of knots (Fáry was a Hungarian mathematician who independently proved the same theorem at the same time as Milnor; see [3]), that has become such a classic result to be included in several introductory texts on differential geometry (see, e.g., [4]).

Milnor enrolled as a Math major in Princeton University, where he received his A.B. in 1951, and his Ph.D. in Mathematics in 1954, under the supervision of Ralph Fox. His research work was so brilliant that he got a position in Princeton's math faculty in 1953, even before completing his doctorate. He was promoted to professor in 1960, staying in Princeton until 1967. He then moved first to the University of California, Los Angeles, and to the Massachusetts Institute of Technology, before joining the faculty of the Institute for Advanced Study at Princeton in 1970. Finally, in 1989 he was appointed Director of the Institute for Mathematical Sciences at Stony Brook University in New York; he is still there, as Co-Director.

He has received all the major mathematical awards. He was a recipient of the Fields Medal in 1962 (when he was 31 years old, well before the age limit of 40), of the Wolf Prize in 1989, and of the Abel Prize in 2011. He was also awarded all three Steele prizes from the American Mathematical Society: for Seminal Contribution to Research in 1982, for Mathematical Exposition in 2004, and for Lifetime Achievement in 2011.

In the next section I shall discuss Milnor's research; but as suggested above Milnor's work goes well beyond proving theorems. He is a very gifted mathematical writer; his books are masterpieces 
of mathematical expositions, read and enjoyed by students and professors alike. Let me just mention Topology from a differential viewpoint [5], originally published in 1965 and still one of the best entrance points to the field of differential topology, and Dynamics in one complex variable [6], which is the book (of the many published on this subject) that I always suggest to students willing to learn this beautiful topic; but all his books have been and still are very influential. Mind you, they are not easy books; the reader has to work his/her way through them. But Milnor has the uncanny ability of precisely identifying the heart of the subject, and of explaining very clearly why things go as they do. A common (and frustrating) experience when reading mathematical papers is going through a proof, confirming the validity of each single step of the argument, and arriving at the end not having the least idea why that result should hold, what is its real significance. And this means we are missing the main point: as scientists, our aim is not just list a few scattered facts about the (mathematical) world, but to understand why it works as it does. Milnor's writings do exactly this: they aim (and succeed) to bring the reader to a full understanding of the subject matter. As written in the Abel Prize Commitee's statement: "Milnor is a wonderfully gifted expositor of sophisticated mathematics. He has often tackled difficult, cutting-edge subjects, where no account in book form existed. Adding novel insights, he produced a stream of timely yet lasting works of masterly lucidity. Like an inspired musical composer who is also a charismatic performer, John Milnor is both a discoverer and an expositor." [7]

And Jack also is a good guy to be around. He does not try to impress, or to show how good he is; he is more interested in understanding what you are saying, and is always willing to answer your questions. In talks, you can immediately see when something catches his attention. His blue eyes focus on the speaker, and if he asks a question it is always right on the mark but never threatening; his aim is not to point out a fallacy in the presentation, but to truthfully understand what the speaker is saying. And sometimes I have the impression that still now he looks at life with the beautiful wonder of a child. I remember his willingness to try a particularly exotic kind of Japanese cuisine in Kyoto; or his amusement in launching himself in a funny folk dance in Cuernavaca, Mexico. But you do not need to accept my word for this; just look at his smile (see Fig. 1; he is the tall guy on the left) and you will understand what I mean. 


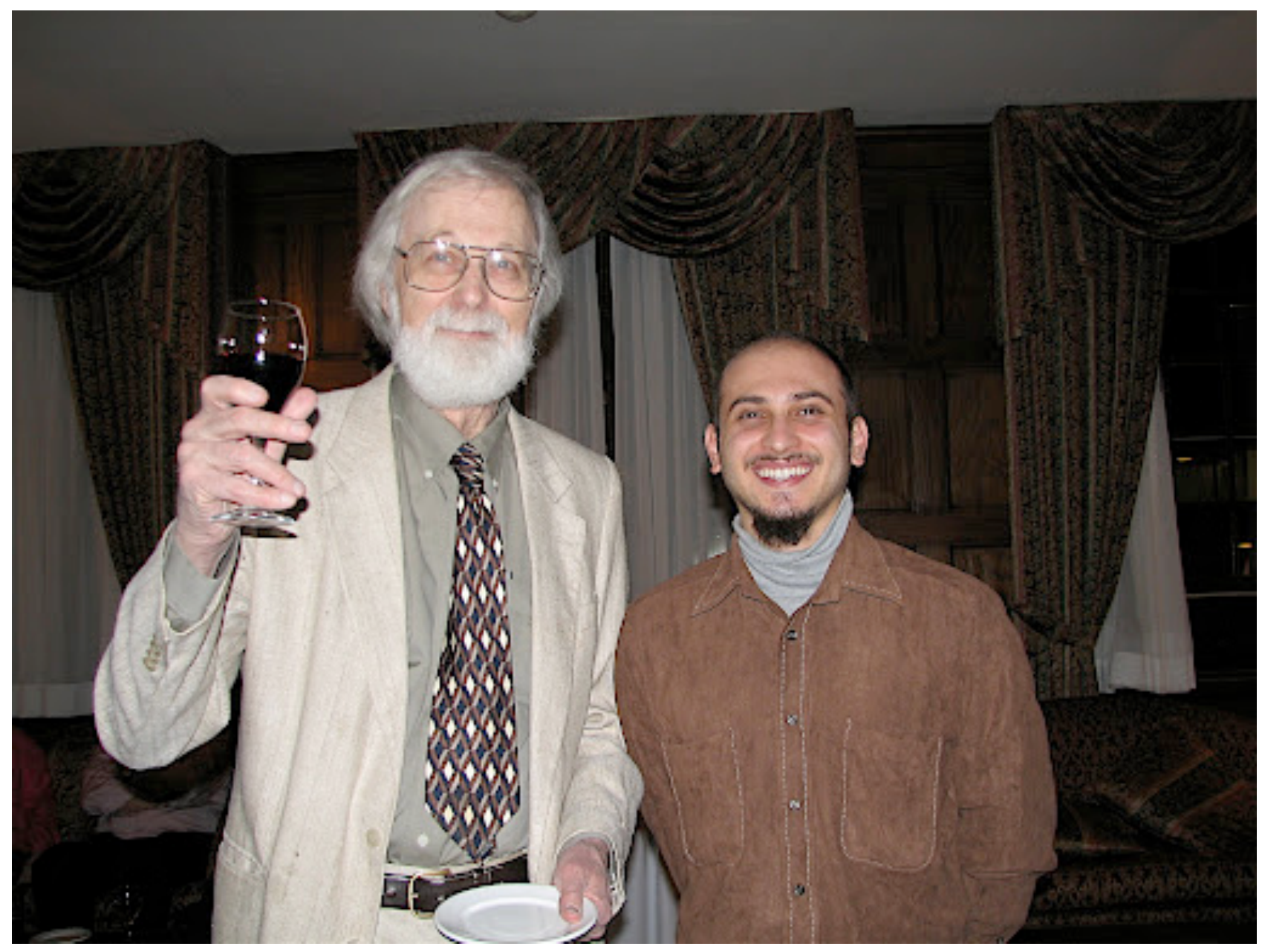

Fig. 1 John Milnor (left) and Enrico Le Donne (photograph courtesy of Enrico Le Donne)

\section{Exotic spheres}

The result I would like to talk about is contained in the famous paper On manifolds homeomorphic to the 7-sphere [8], that by itself started a completely new mathematical subject, differential topology. Let me begin by trying to explain the words in the title.

What is a sphere? To answer this (apparently innocuous) question, we start from the 1dimensional sphere, that is the circumference. Geometrically, the circumference of radius one centered at the origin $O$ in the plane is the set of points at distance 1 from $O$. Introducing Cartesian coordinates centered at $O$, we can represent each point of the plane with a pair $(x, y)$ of real numbers, and Pythagoras' theorem implies that the points of the circumference are exactly the points of the plane represented by pairs $(x, y)$ satisfying the equation

$$
x^{2}+y^{2}=1 \text {. }
$$

Let us then consider the unit sphere in 3-dimensional space, that is the set of points in the space at distance 1 from the origin. Introducing again Cartesian coordinates, each point in space is represented by a triple $(x, y, z)$ of real number, and the points in the unit sphere satisfy the equation

$$
x^{2}+y^{2}+z^{2}=1 \text {. }
$$


The mathematical symbol most often used to denote the unit sphere in 3-dimensional space is $S^{2}$, where $S$ stands for sphere, and the superscript 2 recalls that the sphere is a surface, that is a 2 dimensional object. Analogously, it is customary to denote the unit circumference by the symbol $S^{1}$, to recall that it is a 1-dimensional sphere.

The introduction of Cartesian coordinates allowed us to identify points in the plane with pairs of real numbers, and points in 3-space with triple of real numbers; and the equations describing $S^{1}$ and $S^{2}$ in coordinates clearly had the same structure. Mathematicians cannot help following a pattern, it is part of their nature; and so they immediately define the "3-dimensional sphere $S^{3}$ in a 4dimensional space" as the set of quadruple $(x, y, z, w)$ of real numbers satisfying the equation

$$
x^{2}+y^{2}+z^{2}+w^{2}=1 .
$$

And why stop here? We can as easily define a 4-dimensional sphere in a 5-dimensional space, or a 7-dimensional sphere in a 8-dimensional space; in general, the $n$-dimensional sphere $S^{n}$ in a $(n+1)$ dimensional space is the set of $(n+1)$-uples $\left(x_{0}, \ldots, x_{n}\right)$ of real numbers satisfying the equation

$$
x_{0}^{2}+\ldots+x_{n}^{2}=1 .
$$

This is not only a fatuous trick; the use of coordinates in this way is a basic step for representing and understanding the geometry of the $n$-dimensional analogue of the usual surfaces, called ( $n$ dimensional) manifolds (and so we have introduced another word of the title).

Let me pause a second to justify why it is useful to consider $n$-dimensional objects even if we are only interested in understanding our comfortable 3-dimensional world. Consider a particle in space: to describe its position we just need 3 real numbers, its 3 Cartesian coordinates as described before. But if the particle starts to move, we have to consider its velocity, that can again be represented by using 3 real numbers (its coordinates in a Cartesian system centered at the position of the particle). Thus to describe a moving particle in space we need 6 real numbers - that is we need a 6 dimensional space. And if we want to describe a soccer team of moving particles we need $6 \times 11=66$ real numbers, and we end up in a 66-dimensional space. And if we want to describe an actual soccer team composed by human beings, we need an $N$-dimensional space with $N$ very large: indeed, in the human body there are a lot of parts that can be moved independently, and thus for each player we need at least 6 numbers for each such parts: 6 for each foot, 6 for each knee, 6 for the pelvis, 6 for the torso, 6 for the each hand (not counting the fingers), and so on... But not all $N$-tuples of real numbers can represent a soccer team: there are conditions to be satisfied. For instance, the distance between the left foot and the left knee is constant (it is equal to to the length of the lower left leg); this means that the numbers representing the positions of the left foot and of the left knee must satisfy an equation similar to the ones written above for the spheres. And exactly as happened for 
the sphere, most of these equations (not all, but this is a technical point not worth elaborating on here) define a subset of the $N$-dimensional space which is a high-dimensional analogue of a surface in 3-space; and, in conclusion, to study your favorite soccer team you are forced to use highdimensional manifolds. Think about this next time you turn on the TV to enjoy a soccer match...

We still have one word in the title left to explain: homeomorphic. Roughly speaking, it means "to have the same shape", where here "the same shape" has to be intended in the topological sense (and not in a more geometrical sense that we shall introduce below). Let me explain what it means for the case of surfaces in 3-spaces; very similar things can be said about manifolds in $N$-dimensional space. The idea is that two surfaces in 3-space are homeomorphic if we can deform one into the other continuosly, that is without cutting and without self-intersections. ${ }^{1}$ For instance, a sphere and (the surface of) a rugby ball are homeomorphic: it suffices to inflate the rugby ball (without blowing it up) until it becomes spherical. By the same token, a cube and a sphere are homeomorphic too: again, it suffices to inflate the cube until it loses its edges and becomes round.

However, from another point of view the cube and the sphere geometrically do not have the same shape: the cube has edges, whereas the sphere is smooth everywhere. Being smooth is a identifying characteristic of all manifolds; in particular, the cube is not a manifold (is not a smooth surface). The rugby ball, on the other hand, is; so one can wonder whether the rugby ball and the sphere still have the same shape as smooth manifolds, where we say that two smooth surfaces have the same shape, or, in technical jargon, are diffeomorphic (where "diffeo" here comes from "differentiable", to recall that the smoothness can be expressed in terms of derivatives), if we can deform one onto the other without cutting, self-intersecting and in such a way that all the intermediate surfaces in the deformation are still smooth. ${ }^{2}$

Under this definition, the rugby ball and the sphere are diffeomorphic, because all the intermediate shapes in the inflating process are still smooth. A similar argument also works for much more complicated surfaces, like the one in Fig. 2.a. On the other hand, the doughnut (or, in mathematical terms, the torus) depicted in Fig. 2.b., though smooth, cannot be diffeomorphic to the sphere. Indeed, it is not even homeomorphic to the sphere: there is no way to get rid of that hole without cutting the surface.

On the other hand, one can be hard pressed in finding a smooth surface homeomorphic but not diffeomorphic to the sphere; and indeed it does not exist.

\footnotetext{
1 To be more precise, two surfaces are homeomorphic if there is a continuous bijection between them having a continuous inverse; but allow me to use a slightly imprecise language here for the sake of clarity.

2 Again, the technical definition is more complicated than this: two smooth surfaces are diffeomorphic if there is a differentiable bijection between them having a differentiable inverse.
} 


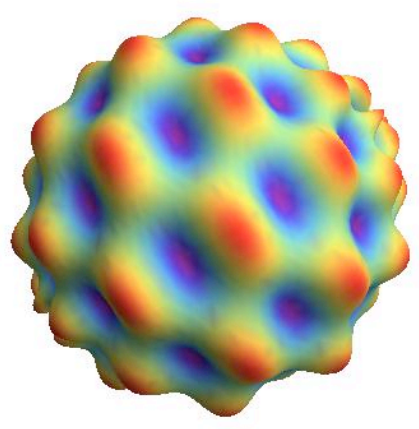

a)

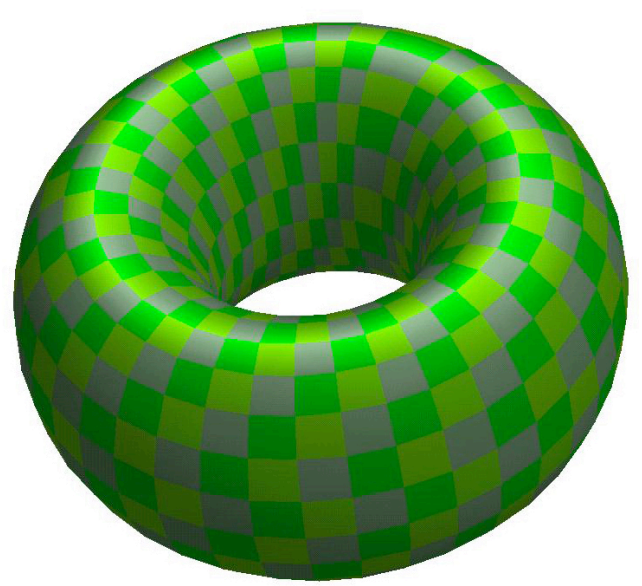

b)

Fig. 2 a) A smooth surface diffeomorphic to the sphere. b) A smooth surface not homeomorphic to the sphere

To explain why, let us try and see what can go wrong (and why it does not). Consider the circumference $S^{1}$ in the plane. An ellipse (like the rugby ball in the 3-space) is clearly diffeomorphic to $S^{1}$, again by inflating. However, this is not the only way to continuously deform an ellipse into a circumference. Indeed, we might first deform the ellipse into a rectangle; then stretch the rectangle until it becomes a square; and then inflate the square until it becomes a circle. If this was the only way we knew for passing from the ellipse to the circumference, we would not be able to conclude that the two are diffeormorphic, because some of the intermediate curves in the deformation were not smooth; the transformation created corners. So the question is: might there exist a smooth curve so complicated (like the one in Fig. 3) to be homeomorphic but not diffeomorphic to a circumference?

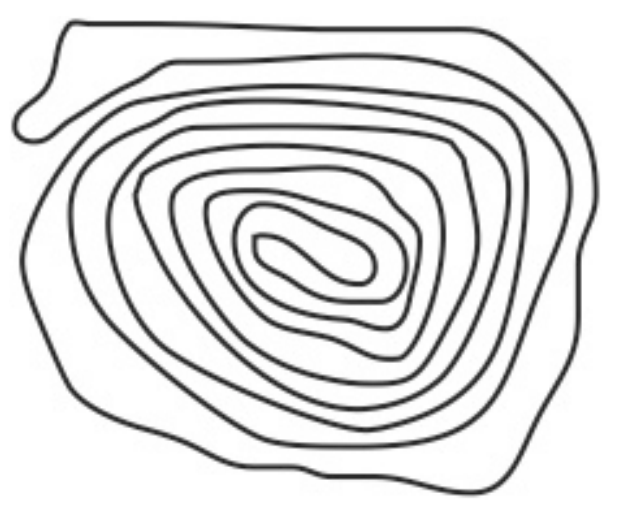

Fig. 3 Is this curve diffeomorphic to a circumference?

Well, no: we can always smooth corners. Indeed, if we have a corner in a curve, we can change the curve just the tiniest bit replacing the corner by a smooth piece; an hyperbola is usually enough (see Fig. 4). 


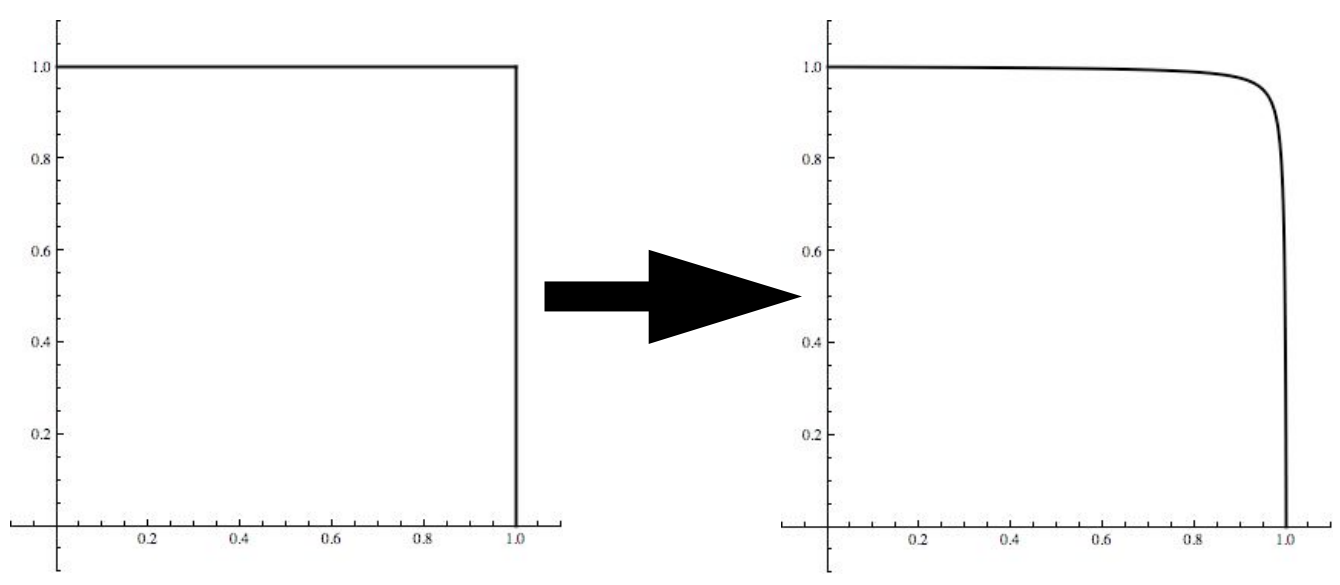

Fig. 4 How to smooth out a corner

So, if during our deformation a corner appears, we can slightly change the deformation by smoothing the corner out just an instant before it appears, obtaining a deformation where all intermediate curves are smooth; and thus any smooth curve homeomorphic to a circumference also is diffeomorphic to the circumference. ${ }^{3}$

The situation is more or less the same for the sphere in 3-space. If deforming a smooth surface (homeomorphic to a sphere) a corner or an edge appears, then we can iron it out, like ironing out creases in a shirt, allowing us to smoothly and safely complete the deformation up to the sphere. In other words, any smooth surface homeomorphic to $S^{2}$ is diffeomorphic to it.

It is then natural to conjecture that this holds in any dimension: any smooth $n$-dimensional manifold homeomorphic to $S^{n}$ is diffeomorphic to it, where $n$ is any positive dimension. This statement is now known as the smooth Poincaré conjecture, because similar in nature to the much more famous topological Poincaré conjecture stating that any $n$-manifold homotopically equivalent to $S^{n}$ is homeomorphic to it, where (very roughly speaking) two manifolds are homotopically equivalent if one can be deformed onto the other without cutting but allowing some identification; for instance, a cylinder is homotopically equivalent to a circumference (just squeeze the cylinder onto a base circumference; this operation does not change the number and nature of the holes, which is what homotopy measures). The topological Poincaré conjecture has been recently settled in the positive by Perelman (see [9] for a popularizing presentation of Perelman's results): an $n$ manifold homotopically equivalent to $S^{n}$ always is homeomorphic to it.

In 1956, when Milnor was working on his paper, the topological Poincaré conjecture was widely open. On the other hand, the smooth Poincaré conjecture was not really a conjecture: most mathematicians took it for granted, without even realizing it; after all, what else can happen? So Milnor's paper came as a complete shock: he showed that a lot more can happen. In dimension 7 ,

\footnotetext{
${ }^{3}$ Of course, this is not a proof; however, a formal proof is not that difficult, and can be understood by third-year math undergraduate students.
} 
there is a 7-manifold which is homeomorphic but not diffeomorphic to a sphere: it has creases that cannot be ironed out, no matter how hard you try (the nightmare of any housewife - or, even more, of any househusband). It is called an exotic sphere; at first glance it looks like a sphere, but becomes positively strange under closer examination. This shows that the topological structure does not completely determine the differentiable (smooth) structure: and so the field of differential topology, the study of the relationships between topology and differentiability, was born.

Milnor's discovery of an exotic 7-sphere is a geometrical masterpiece, based on the most advanced geometrical tools available at the time. Actually, the most difficult part is not building the sphere, but proving that it is exotic. In very rough terms, the construction goes as follow: Milnor divided the standard 7-sphere in two emispheres, a north emisphere and a south emisphere, cutting along the equator. The border of each emisphere is a sphere of one dimension less (think of the usual equator of the standard 2-sphere: it is a circumference, that is a sphere of one less dimension). If we glue together the two emispheres along the respective borders (that is, we identify each point of the border of the north emisphere with exactly one point of the border of the south emisphere and conversely), it is not difficult to see (using a tool called Morse theory; see [10]) that we obtain a manifold still homeomorphic to the 7-sphere. If the identification is well-behaved (for instance, we just rotated one emisphere before gluing it back to the other one), the manifold we obtain is also diffeomorphic to the 7-sphere; but Milnor devised a really wild identification, and when he tried to check whether this new manifold $M$ was still diffeomorphic to the 7-sphere he did not succeed (see [11] for Milnor's recollection of the events). So he decided to try and prove that $M$ was not diffeomorphic to $S^{7}$. A characteristic of the usual 7 -sphere is that it is the boundary of a 8 dimensional ball (exactly as the usual 2-sphere is the boundary of the usual unit 3-dimensional ball, the set of points at distance strictly less than 1 from the origin in 3-dimensional space). Starting from this observation, Milnor managed (and this is the deepest part of his work) to associate to any 7-dimensional manifold a number somehow measuring how far the manifold was from being the boundary of an 8-dimensional manifold; and the association was such that the same number would be assigned to two diffeomorphic manifolds. Since $S^{7}$ is the boundary of the 8-dimensional unit ball, its associated number is 0 . However, the number associated to $M$ is not zero; and hence the only possible conclusion is that $M$ is not diffeomorphic to $S^{7}$, it is an exotic sphere.

This is only the beginning of the story. The mathematical community, as soon as it started to recover from the shock of discovering that something given for granted was actually false, realized the potentialities and the importance of the new field of differential topology, and started asking questions. For instance, now that we know that there might be smooth $n$-manifolds homeomorphic but not diffeomorphic to the $n$-sphere, is it possible to count them? That is, is it possible to exactly 
say how many $n$-manifolds not diffeomorphic to each other but all homeomorphic to the $n$-sphere there are for each dimension $n$ ?

Let us called $a_{n}$ this number. In 1963, Milnor and Kervaire [12] showed that $a_{7}=28$, that is that there are exactly 28 different 7-manifolds homeomorphic to the 7-sphere. They can be obtained as the set of 5-tuples $\left(z_{0}, z_{1}, z_{2}, z_{3}, z_{4}\right)$ of complex numbers satisfying the following equations

$$
\left|z_{0}\right|^{2}+\left|z_{1}\right|^{2}+\left|z_{2}\right|^{2}+\left|z_{3}\right|^{2}+\left|z_{4}\right|^{2}=1,\left(z_{0}\right)^{3}+\left(z_{1}\right)^{6 k-1}+\left(z_{2}\right)^{2}+\left(z_{3}\right)^{2}+\left(z_{4}\right)^{2}=0
$$

for $k$ going from 1 to 28 .

The quest for solving this problem for all $n$ has been going on since then, and it is not finished yet. Thanks to the work of many people (let me just quote Browder [13] and Kervaire-Hill-Ravenel [14], but many more names should be added; see $[15,16]$ for recent surveys) ways to compute $a_{n}$, at least in principle, have been devised. In particular, $a_{n}$ is now known for all small values of $n$ (at least for $n \leq 64$; see Fig. 5 for a table containing its first few values), with one surprising (and frustrating) exception: $n=4$. Indeed, the 4-dimensional case is completely open, to the point that it is conjectured that there are infinitely many exotic 4-spheres, but till now nobody has been able to prove the existence of a single one! So after more than 55 years Milnor's discovery has not yet finished to reveal all its intricacies.

\begin{tabular}{|l|l|l|l|l|l|l|l|l|l|l|l|l|l|l|l|l|l|l|l|l|}
\hline$n$ & 1 & 2 & 3 & 4 & 5 & 6 & 7 & 8 & 9 & 10 & 11 & 12 & 13 & 14 & 15 & 16 & 17 & 18 & 19 & 20 \\
\hline$a_{n}$ & 1 & 1 & 1 & $?$ & 1 & 1 & 28 & 2 & 8 & 6 & 992 & 1 & 3 & 2 & 16436 & 4 & 16 & 16 & 261632 & 24 \\
\hline
\end{tabular}

Fig. 5 The first few values of $a_{n}$

\section{References}

[1] Milnor, J.W.: On the total curvature of knots. Ann. of Math. 52 (1950), 248-257.

[2] Abate, M.: The many faces of Lorenz knots. In Imagine Math, M. Emmer ed., Springer Italia, Milano, 2012, pp. 169-174.

[3] Fáry, M.I.: Sur la courbure totale d'une courbe gauche faisant un nœud. Bull. Soc. Math. France 77 (1949), 128-138.

[4] do Carmo, M.P.: Differential geometry of curves and surfaces. Prentice-Hall, Inc., Englewood Cliffs, N.J., 1976.

[5] Milnor, J.W.: Topology from the differentiable viewpoint. Revised reprint of the 1965 original. Princeton Landmarks in Mathematics. Princeton University Press, Princeton, NJ, 1997. 
[6] Milnor, J.W.: Dynamics in one complex variable. Annals of Mathematics Studies, 160. Princeton University Press, Princeton, NJ, 2006.

[7] http://www.abelprize.no/c53720/binfil/download.php?tid=53552

[8] Milnor, J.W.: On manifolds homeomorphic to the 7-sphere. Ann. of Math. 64 (1956), 399-405.

[9] Cipra, B.: First of seven millennium problems nears completion. In What's happening in the mathematical sciences, Vol. 6, D. Mackenzie, B. Cipra eds., American Mathematical Society, Providence, RI, 2006, pp. 3-13.

[10] Milnor, J.W.: Morse theory. Annals of Mathematics Studies, No. 51. Princeton, NJ: Princeton University Press, 1963.

[11] Milnor, J.W.: Classification of (n-1)-connected $2 n$-dimensional manifolds and the discovery of exotic spheres. In Surveys on surgery theory, Vol. 1 Ann. of Math. Stud., 145, Princeton Univ. Press, Princeton, NJ, 2000, pp. 25-30.

[12] Milnor, J.W., Kervaire M.A.: Groups of homotopy spheres: I. Ann. of Math. 77 (1963), 504537.

[13] Browder, W.: The Kervaire invariant of framed manifolds and its generalization. Ann. of Math. 90 (1969), 157-186.

[14] Hill, M.A., Hopkins, M.J. , Ravenel, D.C.: On the non-existence of elements of Kervaire invariant one. Preprint, arXiv:0908.3724, 2009.

[15] Milnor, J.W: Differential topology forty-six years later. Notice Am. Math. Soc. 58 (2011), 804-809.

[16] Mackenzie, D.: Aso ne heroic age ends, a new one begins. In What's happening in the mathematical sciences, Vol. 8. D. Mackenzie ed., American Mathematical Society, Providence, RI, 2011, pp. 118-129. 